\title{
La desigualdad entre hombres y mujeres en la antigua Roma: un estudio sobre sus concepciones y conocimiento en el alumnado de historia
}

Inequality between men and women in ancient Rome: a study about its conceptions and knowledge in history students

\section{Daniel Mateo Corredor}

Universidad de Alicante.

E-mail: daniel.mateo@ua.es

Orcid ID: ORCID ID: 0000-0002-9624-2II3

\section{María Pastor Quiles}

Universidad de Alicante.

E-mail: m.pastor@ua.es

Orcid ID: ORCID ID: 0000-000|-6||2-6422

Resumen: Este trabajo de investigación realiza un acercamiento a las concepciones y al conocimiento del estudiantado de Historia acerca de diferentes aspectos relacionados con la desigualdad entre sexos en la antigua Roma. Mediante el diseño y la aplicación de un cuestionario con una escala de valoración tipo Likert, analizamos las respuestas del alumnado en torno a distintas afirmaciones que abordan temas como la desigualdad en el acceso al poder político, los derechos jurídicos o las actividades económicas de las mujeres en época romana. Las respuestas obtenidas parecen reflejar que estos son aspectos poco abordados en los contenidos universitarios acerca de la Historia de Roma, caracterizados tradicionalmente por un sesgo androcéntrico, como los de otras muchas áreas de conocimiento. Estos resultados también evidencian la necesidad de continuar trabajando en el desarrollo del conocimiento acerca de la desigualdad histórica entre mujeres y hombres, así como en su incorporación en los planes de estudio.

Palabras Clave: Historia. Educación. Alumnado. Desigualdad. Género. Roma. Antigüedad. Patriarcado.

Abstract: This research study aims to approach the conceptions and knowledge of 
History students about different aspects in relation to sexual inequality in ancient Rome. By means of the elaboration and implementation of a questionnaire based on a Likert rating scale, the reactions of the students are analysed. The propositions offered to the students address aspects such as inequality in the access to political power structures, legal rights or the economic activities developed by women in the Roman period. The student's responses reveal that these are aspects barely included in the university programmes on ancient Rome, which have been traditionally characterised by an androcentric bias as it occurs also in many other areas of knowledge.

These results also highlight the necessity to continue developing knowledge about the historic inequality between women and men and also of the incorporation of this knowledge in the study programmes.

Keywords: History. Education. Students. Inequality. Gender. Rome. Antiquity. Patriarchy.

\section{INTRODUCCIÓN}

$\square$

L presente trabajo se enmarca en un proyecto de investigación centrado en el análisis de la percepción social y el conocimiento del alumnado del Grado en Historia de la Universidad de Alicante acerca de la desigualdad entre hombres y mujeres en el pasado, concretamente, durante la Prehistoria y la Antigüedad (Pastor Quiles y Mateo Corredor, 2018). En este texto presentamos los resultados y el análisis de las cuestiones planteadas al estudiantado referidas a la época romana.

Las fuentes históricas disponibles para conocer el periodo romano son amplias y variadas. Además, la época romana ocupa un lugar central en los planes de estudio universitarios de Historia en muchos países. El interés suscitado por la antigua Roma es muy grande a muchos niveles, desde en la propia investigación, hasta en la difusión del conocimiento, con una posición central de esta temática en museos, itinerarios turísticos arqueológicos y en la divulgación de la Historia. No solo el grado de conocimiento acerca de la Roma antigua es elevado en lo que se refiere a la propia academia, sino que también puede considerarse relativamente alto el conocimiento que una parte de la sociedad, externa al mundo académico, posee acerca de este periodo histórico. No obstante, existen cuestiones fundamentales de la sociedad romana antigua que son prácticamente desconocidas para la gran mayoría de la sociedad. Este fenómeno no es exclusivo de la Antigüedad o del periodo romano, dándose también en el caso de otras cronologías y campos de estudio. Entre estas cuestiones menos visibilizadas están las que abordamos en este trabajo, referidas a la desigualdad entre mujeres y hombres.

La sociedad romana se caracterizó por una enorme desigualdad entre hombres y mujeres, desigualdad cuyas características y consecuencias dependían también de otros factores como, por ejemplo, la clase social (López Medina, 2008). Y como ocurre también para otros periodos históricos, queda camino por transitar en cuanto a su investigación, pero, sobre todo, en lo que se refiere a la recepción e incorporación de los resultados al conocimiento androcéntrico mayoritariamente establecido y reconocido 
-con lo que ello supone en cuanto a su cuestionamiento a nivel estructural-y, en el ámbito que nos ocupa, al conocimiento transmitido en los planes de estudio universitarios.

Conocer las concepciones del alumnado de Historia acerca de estas cuestiones es enormemente interesante debido al tradicional androcentrismo de la disciplina y de sus contenidos, a pesar de los significativos cambios que se han ido produciendo en los últimos años, tanto a nivel de investigación ${ }^{1}$, como en los planes de estudio. Es importante tener en cuenta que entre las competencias generales del título de Grado en Historia de la Universidad de Alicante se incluye el «compromiso social con la pluralidad y diversidad de realidades de la sociedad actual, desde el respeto a los derechos fundamentales y a la igualdad entre hombres y mujeres (...)». Asimismo, en los planes de estudio del actual Grado en Historia, cabe resaltar la incorporación oficial de otras cuestiones abordadas en este trabajo de investigación. Así, entre las competencias específicas de la titulación se incluye «emplear con propiedad los conceptos, categorías, teorías y temas más relevantes de las diferentes ramas de la investigación histórica (económica, social, política, cultural, de género, entre otras) (...)». De este modo, en algunas asignaturas del Grado en Historia de la Universidad de Alicante se han incorporado contenidos con perspectiva de género².

El principal objetivo de este trabajo es conocer las respuestas y orientaciones del alumnado acerca de las proposiciones concretas sobre la sociedad romana antigua que más adelante se presentan. Además, se evalúa si se producen diferencias significativas en las respuestas en función del sexo y la edad de los alumnos y alumnas, así como entre quienes están finalizando los estudios del Grado en Historia respecto a quienes acaban de comenzarlos. Este proyecto nace del interés por conocer los conocimientos adquiridos y los puntos de vista que sobre este tema poseen los futuros historiadores e historiadoras, siendo este colectivo clave por muchas razones. Sin duda, la relevancia de conocer las visiones del alumnado de Historia acerca de la desigualdad entre sexos viene en buena parte motivada por su papel como principal transmisor de conocimientos y valoraciones sobre ella, sobre todo, aunque no exclusivamente, mediante el ejercicio futuro de la enseñanza de la Historia en los diferentes niveles académicos y, en especial, en la enseñanza secundaria. Por otro lado, la necesidad de continuar abordando la desigualdad entre sexos en relación a la Historia como disciplina se debe también a la enorme importancia del análisis histórico de esta desigualdad para la transformación de la sociedad.

1 Cabe resaltar el reciente auge de los estudios de género sobre la Antigüedad. Para un estado de la cuestión sobre la aplicación de la perspectiva de género al análisis de la antigua Roma, véase Conesa Navarro (2016) y para el ámbito de la Antigüedad en general véase Cid López (2015a).

2 Como puede verse en las guías docentes y en los datos recogidos por Mónica Moreno Seco (2014). Además, estos cambios también se están introduciendo en los estudios de Máster. Así, centrándonos en la Antigüedad, en el Máster en «Historia del Mundo Mediterráneo y sus Regiones. De la Prehitoria a la Edad Media», de reciente creación, se incluye una lección sobre «Las mujeres en el Mediterráneo antiguo». Asimismo, en los últimos años los temas con perspectiva de género son uno de los campos de estudio más elegidos en Trabajos de Fin de Grado y Trabajos de Fin de Máster. 


\section{METODOLOGÍA}

La población de este estudio está compuesta por el alumnado del Grado en Historia de la Universidad de Alicante. La muestra se ha seleccionado de manera no aleatoria y no estratificada, siendo el criterio de inclusión que el alumnado se encontrara cursando primer o cuarto curso, ya fuese en los grupos de mañana o tarde. Esta selección se realizó con el fin de poder comparar las diferencias entre el alumnado que llevase poco tiempo cursando la carrera y aquel que estaba terminando el último curso, uno de los objetivos específicos de esta investigación. La muestra está formada por 108 alumnas y alumnos, de los que 60 pertenecían a primer curso y 48 a cuarto curso. Por sexos, la muestra incluye a 64 alumnos y 44 alumnas. La mayor parte del alumnado posee entre 18 y 24 años, si bien un pequeño porcentaje -17,6\% - supera esa edad, encontrándose la mayoría entre 25 y 30 años y teniendo solo siete más de 30 años, con 64 el mayor de ellos (Figura 1).

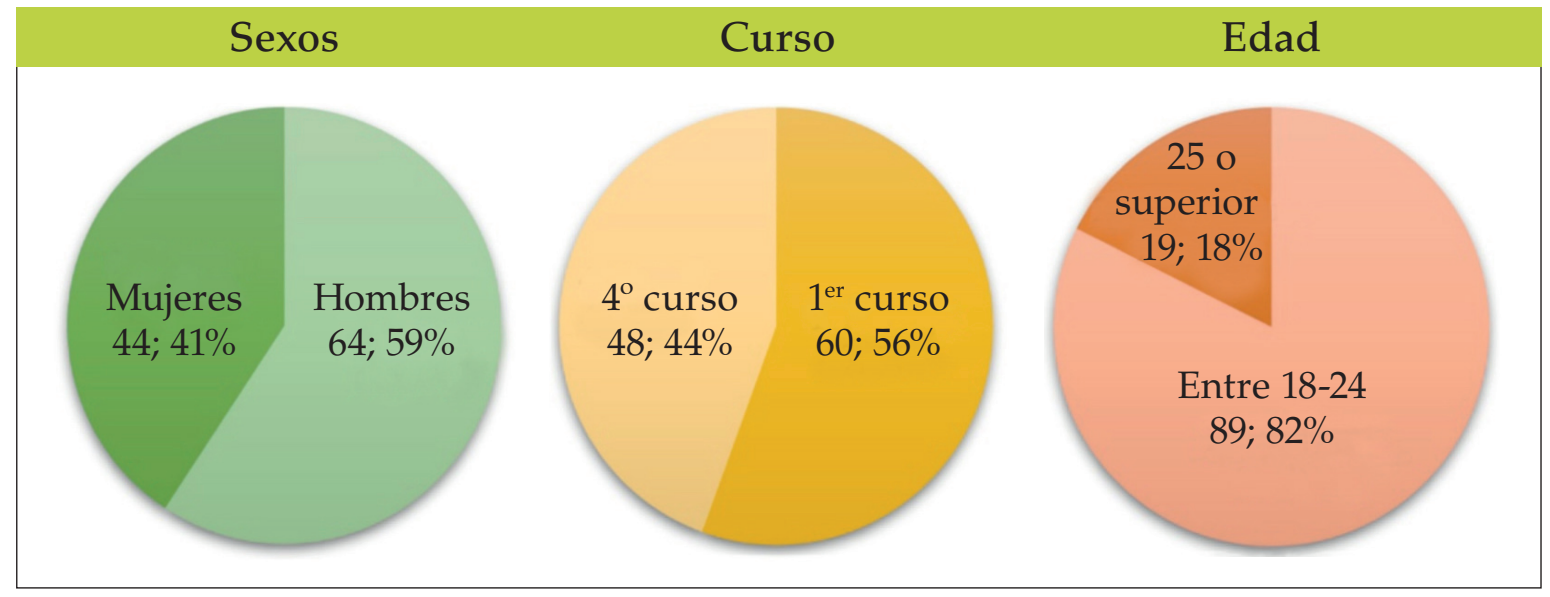

Figura 1

Análisis por categorías de la muestra $(\mathrm{n}=108)$ (elaboración propia)

El método utilizado para obtener la información fue un cuestionario cerrado con una escala de valoración tipo Likert. Se trata de una escala aditiva con un nivel ordinal fundamentada en la reacción del sujeto a una serie de ítems o proposiciones y que posibilita medir actitudes y conocer el nivel de conformidad, gracias al grado de acuerdo o desacuerdo del sujeto con cada ítem o proposición (Namakforoosh, 2000). Acada respuesta se le da una puntuación, obteniendo tras la suma de las respuestas de cada sujeto, una puntuación total que muestra su posición respecto al objeto analizado. Su elaboración es más sencilla que otras escalas y tiende a alcanzar una alta fiabilidad (Farrell et al., 1985). Sin duda, es un instrumento muy utilizado por las ciencias sociales y humanísticas, por ejemplo, para identificar las percepciones de los y las estudiantes en el ámbito educativo, incluyendo la evaluación de la actividad docente (Sierra Bravo, 2001; Fabila Echauri et al., 2013). Este tipo de cuestionario nos ha permitido valorar las reacciones hacia cada una de las afirmaciones del mismo, así como realizar una aproximación global a las respuestas. 
En nuestro estudio escogimos una escala de estimación de cinco niveles para cada ítem del cuestionario, de acuerdo a las siguientes alternativas de respuesta presentadas de manera horizontal: 1 (totalmente en desacuerdo); 2 (en desacuerdo); 3 (ni de acuerdo ni en desacuerdo); 4 (de acuerdo); 5 (totalmente de acuerdo). Asimismo, optamos por incluir afirmaciones con direcciones en sentido positivo y negativo, en aras de limitar la tendencia a contestar afirmativamente, uno de los sesgos de la escala Likert. De igual modo, los ítems de cada uno de los temas se presentaron en el cuestionario de manera no agrupada, excepto los relativos a la valoración de la representación de las mujeres en los contenidos del Grado de Historia, que se entregaron únicamente al alumnado de cuarto curso y que ya han sido objeto de análisis (Pastor Quiles y Mateo Corredor, 2018).

Tras su diseño inicial, el cuestionario se presentó a diversos especialistas y se realizó a un grupo piloto ${ }^{3}$, lo que hizo posible eliminar 12 ítems y ajustar los seleccionados en contenido y forma, mejorando así la validez del instrumento. Se calculó el alfa de Cronbach para el conjunto de ítems con el fin de estimar su fiabilidad, obteniendo $\alpha=0,793$, lo que indica una consistencia interna aceptable (George y Mallery, 2005: 231). El cuestionario final está formado por un total de 32 proposiciones comunes a los grupos de primero y cuarto curso, con un tiempo de aplicación de 10-15 minutos aproximadamente.

Acudimos a las aulas en horario lectivo al final del segundo semestre del curso 2016-17 y presentamos el cuestionario a los estudiantes, con la amable colaboración del profesorado ${ }^{4}$. De un total de 219 alumnos y alumnas matriculados en primer curso y de 109 en cuarto curso, realizaron la prueba $60-27,4 \%$ - y $48-44 \%$ - estudiantes, respectivamente. Los cuestionarios fueron rellenados de manera anónima, animando al alumnado a que contestara con sinceridad y solicitando que nos proporcionara sus datos de sexo y edad, que junto al curso constituyen las variables independientes. Una vez finalizada la fase de aplicación del instrumento, se procedió a la codificación y al tratamiento cuantitativo de los datos, sometidos a un análisis estadístico con el uso de medidas de tendencia central, de dispersión, frecuencias y porcentajes.

Las proposiciones del cuestionario están estructuradas en diferentes temas, centrándonos en el presente texto en los 11 ítems que se refieren a los aspectos relacionados con la desigualdad durante la antigua sociedad romana. Acerca de estas proposiciones, es importante tener en cuenta que se trata de formulaciones necesariamente generales, pensadas para aproximarnos a las concepciones del alumnado y

\footnotetext{
3 Queremos dar las gracias a todas las personas que nos prestaron su colaboración contestando al cuestionario piloto, así como al personal investigador que nos asesoró en la elaboración del cuestionario definitivo y nos apoyó en su puesta en práctica. Por supuesto, los errores y omisiones son responsbilidad nuestra.

4 Agradecemos a las alumnas y alumnos de estos grupos su generosa disposición y participación, sin las que este proyecto no hubiese sido posible.
} 
explorar las respuestas, que poder mesurar también de forma cuantitativa. En ningún caso las afirmaciones pueden reflejar toda la complejidad de los procesos históricos ni su evolución a lo largo de los siglos de historia de la antigua Roma. Las ideas a valorar por el alumnado son formulaciones de diversa naturaleza diseñadas para el registro y estudio de sus respuestas y no suponen literalmente ni argumentaciones resultado de la investigación histórica ni opiniones de los autores.

\section{RESULTADOS}

En este apartado se recogen las proposiciones acerca de la Historia de Roma presentadas a los alumnos y alumnas y los resultados obtenidos. Optamos por presentar estos ítems de manera agrupada en función del tema, en lugar de mantener su orden original en el cuestionario, en el que se alternaban las proposiciones acerca de los diferentes aspectos abordados. El alumnado encuestado, o bien se encuentra en el primer curso de la titulación y ha tenido, apenas, un contacto inicial con la antigua Roma, o está ya en cuarto curso. En este último caso, dicho estudiantado ya ha cursado los contenidos establecidos en el Grado en Historia acerca de este periodo. No obstante, puede considerarse que los contenidos universitarios de Historia de Roma, con una importante tradición androcéntrica, no han enfatizado el estudio la desigualdad entre hombres y mujeres en la sociedad romana, a pesar de que es bien conocido que este fenómeno impregna claramente este periodo, al igual que la Antigüedad en general. Del mismo modo, la enseñanza de la Historia Antigua en la educación secundaria se ha caracterizado también por este importante androcentrismo, acentuado por una continuidad en cuanto a los contenidos desde inicios de la década de 1990, a pesar de los cambios producidos en la forma (García Santa María y Pagès Blanch, 2008).

Estos y otros factores podrían influir en las respuestas del alumnado ante las cuestiones planteadas, al igual que la visión que posee la sociedad en general sobre la Antigüedad romana. La antigua Roma ocupa un lugar muy importante en los recursos culturales y de ocio, como la novela histórica, las series de televisión y, sobre todo, el cine. El número de producciones cinematográficas ambientadas en la sociedad romana antigua es muy considerable, habiéndose convertido algunos de estos títulos en grandes clásicos del cine (Serrano Lozano, 2012). Y, a propósito de la perspectiva androcéntrica de la Historia Antigua mencionada, cabe poner de manifiesto su vigencia en estos recursos no académicos, que generan poderosas imágenes sobre el pasado en la sociedad. Por ejemplo, entre los múltiples ejemplos generados para la pantalla desde inicios de los años 90 -que pueden haber generado referencias en el alumnado con una probabilidad mayor que los producidos con anterioridad-, apenas encontramos un par de personajes femeninos protagonistas destacados ${ }^{5}$, entre las producciones ambientadas en la Antigüedad, dirigidas tanto a público infantil como adulto.

\footnotetext{
5 Xena, la princesa guerrera (1995-2001), protagonista de una serie de aventuras y fantasía ambientada en la antigua Grecia, e Hipatia de Alejandría, protagonista de la película Ágora (2009), basada en esta misma científica del Egipto del sigloV d. C.
} 


\subsection{Percepción y conocimiento acerca de la desigualdad política y jurídica entre hombres y mujeres en la antigua Roma}

Como punto de partida, de las cuestiones propuestas en el marco de este proyecto abordadas en un trabajo anterior (Pastor Quiles y Mateo Corredor, 2018), queremos traer a colación solo una de ellas, directamente relacionada con el mundo romano y con las proposiciones del cuestionario que presentamos aquí. El ítem al que nos referimos es el que nos permitió conocer que más del $81 \%$ el alumnado encuestado del Grado en Historia afirmó estar de acuerdo o muy de acuerdo en aceptar el carácter patriarcal de la sociedad romana antigua. Esta afirmación alcanza un promedio de 4,3 puntos, sin que se hayan observado apenas diferencias entre las respuestas de hombres y mujeres, siendo reconocido con mayor contundencia $-4,6$ puntos- entre el alumnado de cuarto curso, respecto al de primero $-4,1$ puntos-.

Considerado esto, presentamos los resultados de este estudio respecto a diferentes afirmaciones relacionadas con la desigualdad entre sexos en época romana en diferentes ámbitos, como la política o el derecho (Figura 2).

El primero de los ítems analizados afirma que el pater familias tenía derecho sobre la vida de su esposa en los primeros siglos de la República romana. A partir de la información transmitida en las fuentes clásicas se tiende a aceptar la aplicación del ius vitae et necis sobre la mujer sujeta a la manus de su esposo (Pomeroy, 1995: 150, 153; Fayer, 2005: 207; Benke, 2012; Urbanik, 2016), al igual que sobre el resto de miembros de la familia sujetos a la patria potestas (Dion Hal. Ant. Rom. 2, 26-27). Ante la frase propuesta, una parte mayoritaria del alumnado del Grado en Historia se ha manifestado de acuerdo o muy de acuerdo, con el 42,6\% y el 15,7\% respectivamente, alcanzando un promedio del 3,4. Apenas observamos una décima de variación comparando el promedio entre sexos y entre cursos, siendo dos décimas mayor el promedio en función de la edad, con un 3,6 para el grupo de 25 años o superior, que se muestra ligeramente más de acuerdo.

De igual modo, en el alumnado encuestado se revela una tendencia a validar la proposición de que la función principal que se otorgaba socialmente a las mujeres de la oligarquía romana era la reproducción, como ha sido ampliamente señalado por la investigación (Pomeroy, 1995: 164; López Medina, 2008: 222, 238; Casamayor Mancisidor, 2015a: 8) y de la que se derivaba precisamente el elevado estatus social de las matronas romanas. Así, el 41,7\% se muestra de acuerdo y el 10,2\% muy de acuerdo, aunque también es reseñable que el 26,9\% se manifiesta en desacuerdo. Ante esta afirmación destaca la gran diferencia, de casi 1 punto, que encontramos entre sexos, con un promedio del 2,9 en el caso de los alumnos varones, mientras que las alumnas se muestran notablemente más de acuerdo con la afirmación, con 3,8 puntos. Asimismo, el alumnado de cuarto curso presenta un promedio dos décimas superior al de primero, con un 3,4 y un 3,2 respectivamente. 


\section{Desigualdad política y jurídica entre hombres y mujeres en la antigua Roma}

1. En los primeros siglos de la antigua Roma, legalmente el «pater familias» tenía derecho a decidir sobre la vida o la muerte de su esposa.

2. La función principal que se atribuía a las mujeres de la elite social romana era la reproducción.

3. Las mujeres estaban excluidas de las votaciones políticas celebradas en la antigua República romana.

4. Las instituciones educativas romanas estaban reservadas exclusivamente a los hombres.

5. El Imperio Romano nunca tuvo una mujer en el cargo de emperador.

6. Legalmente, las mujeres podían divorciarse en la antigua Roma.
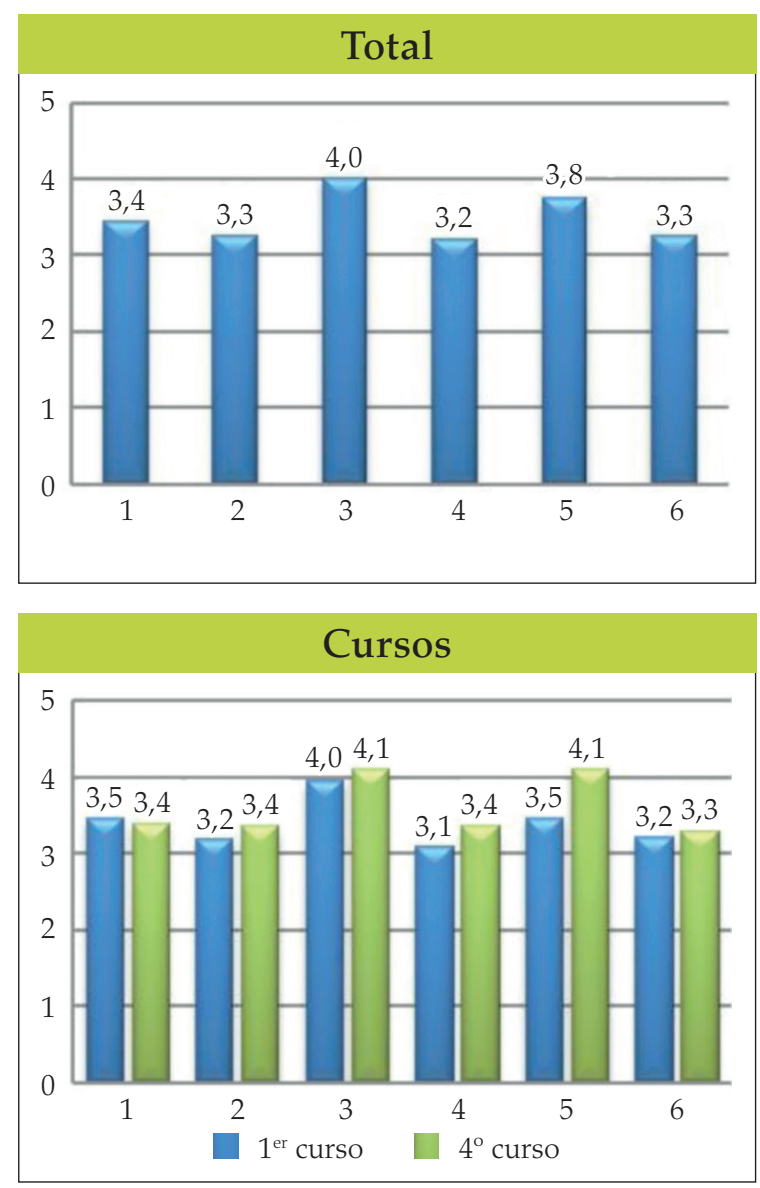

\begin{tabular}{|l|l|l|l|l|}
\hline 1-TED & 2-ED & $\begin{array}{c}\text { 3-Ni DA } \\
\text { ni ED }\end{array}$ & $4-\mathrm{DA}$ & $5-\mathrm{TDA}$ \\
\hline $\mathbf{8}, 3 \%$ & $13,9 \%$ & $19,4 \%$ & $42,6 \%$ & $15,7 \%$ \\
\hline $\mathbf{4}, 6 \%$ & $26,9 \%$ & $16,7 \%$ & $41,7 \%$ & $10,2 \%$ \\
\hline $4,6 \%$ & $4,6 \%$ & $5,6 \%$ & $54,6 \%$ & $30,6 \%$ \\
\hline $7,4 \%$ & $24,1 \%$ & $17,6 \%$ & $42,6 \%$ & $8,3 \%$ \\
\hline $3,7 \%$ & $13,9 \%$ & $14,8 \%$ & $38,9 \%$ & $28,7 \%$ \\
\hline $2,8 \%$ & $23,1 \%$ & $27,8 \%$ & $38,9 \%$ & $7,4 \%$ \\
\hline
\end{tabular}
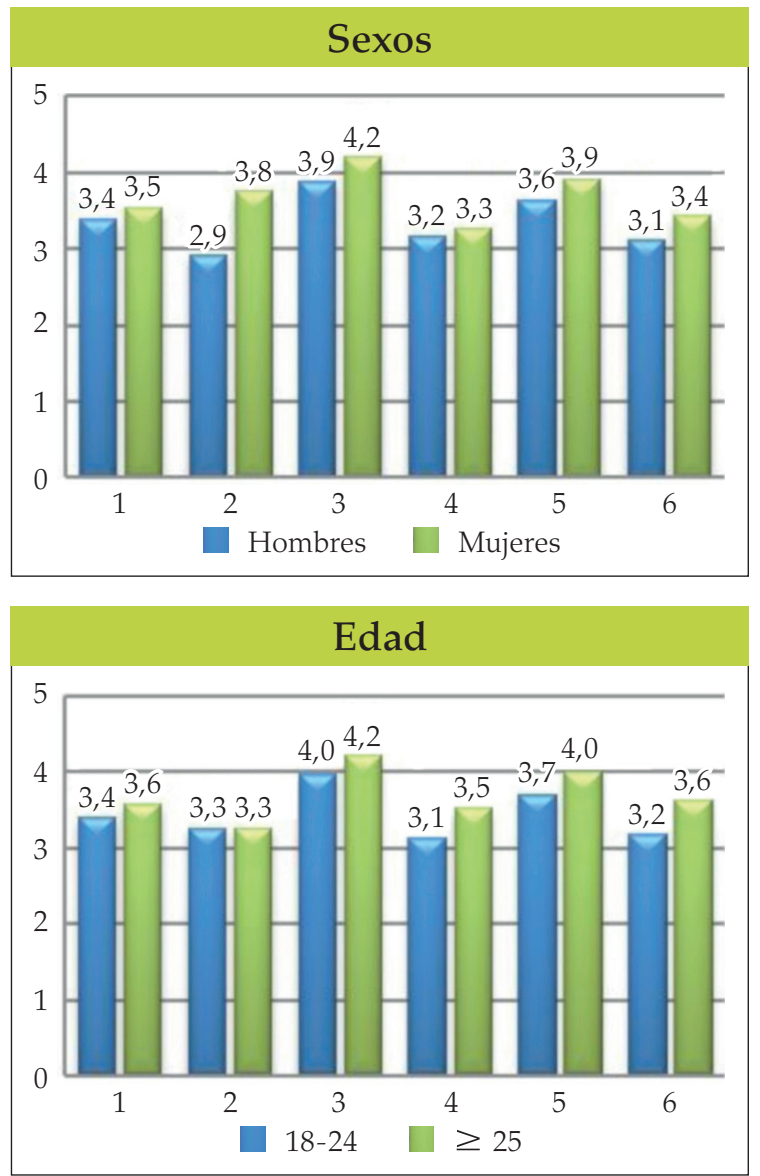

Figura 2

Ítems con porcentajes de respuesta y promedio por categorías (elaboración propia) 
La afirmación «Las mujeres estaban excluidas de las votaciones políticas celebradas en la antigua República romana» recibe la mayor aceptación de todos los ítems abordados en este trabajo. Así, la mayor parte del alumnado es consciente de que la marginación de las mujeres del ámbito político a lo largo de casi toda la Historia también se produjo en época romana, donde las ciudadanas romanas carecían del ius honorum y del ius suffragii (D. 50, 17, 2. pr. [Ulp. 1 Sab.]), más allá de la influencia que pudiesen ejercer y de su posible participación en determinadas asambleas (Bauman, 1992: 60; Bravo Bosch, 2011: 18; Castán Pérez-Gómez, 2013). En esta línea, un 30,6\% de los alumnos y alumnas se muestra muy de acuerdo, y un 54,6\% de acuerdo, con un promedio total de 4 . Ante esta proposición, las mayores diferencias las encontramos de nuevo en una comparación de las respuestas entre sexos, con un 3,9 de promedio en los hombres y un 4,3 en las mujeres.

En época romana, si exceptuamos el periodo monárquico y los inicios de la República, se permitía el acceso de determinadas mujeres romanas a la educación (Hemelrijk, 1999; Cid, 2001), aunque esta educación fuera distinta para hombres y mujeres (Pomeroy, 1995: 170; López Medina, 2008: 245). Aunque con una mayor dispersión que en el anterior ítem, en el alumnado hay una notable tendencia a aceptar la afirmación de que las instituciones educativas en época romana estaban reservadas en exclusiva a los hombres. Así, un 31,5\% se manifiesta totalmente en desacuerdo o en desacuerdo, mientras que el $50,9 \%$ se posiciona de acuerdo o muy de acuerdo. Con un promedio total del 3,2, el alumnado de cuarto curso alcanza el 3,4 y el de primero se queda en el 3,1, mientras que por grupos de edad la diferencia es de 4 décimas, siendo el promedio más alto el del grupo de mayor edad, con un 3,5. En este caso, vemos cómo el hecho de haber cursado el Grado de Historia no se corresponde con dar una respuesta que podríamos considerar más acertada.

Por otro lado, el cargo de emperador siempre estuvo reservado a hombres. No obstante, ante el ítem que negaba la presencia de mujeres en la máxima jefatura del imperio, cabe resaltar que, aunque un total del 65,6\% del estudiantado afirma estar de acuerdo o muy de acuerdo, un nada desdeñable 13,9\% se sitúa en desacuerdo y un $3,7 \%$ totalmente en desacuerdo. También puede sorprender que un 14,8\% opte por la posición intermedia, «ni de acuerdo ni en desacuerdo». En conjunto, se alcanza un promedio del 3,8, registrándose una diferencia de 3 décimas entre hombres y mujeres, con un 3,6 y un 3,9 respectivamente, posicionándose las alumnas más en desacuerdo. Aunque, sobre todo, destaca que la puntuación favorable a esta afirmación es 6 décimas superior entre el alumnado de cuarto curso respecto a los de primero, con un 4,1 y un 3,5 respectivamente, mostrándose más de acuerdo el alumnado de cuarto que el de primero con la exclusión de las mujeres del cargo de emperador, tal y como cabría esperar.

El ítem que suscita mayor diversidad en las respuestas es el que afirma que las mujeres podían divorciarse en época romana, tal y como evidencian las fuentes clásicas a partir época clásica, al margen de que, en la práctica, la posibilidad de llevar adelante un divorcio hubiera recaído en los hombres en la mayoría de los casos, 
enespecial hasta las últimas décadas de la República y el periodo augusteo (Salisbury, 2001: 305; Levick, 2012: 99; Cantarella, 2016; entre otros). Ante esta afirmación, observamos que se da el mayor número de respuestas «ni de acuerdo ni en desacuerdo» en este estudio, con el $27,8 \%$. Además, aunque existe una mayor tendencia a aceptarlo, con un $38,9 \%$ del alumnado mostrándose de acuerdo y un 7,4\% muy de acuerdo, el porcentaje del alumnado que se sitúa en desacuerdo o totalmente en desacuerdo alcanza el 26,9\%. Con un promedio total del 3,3, es reseñable que la puntuación obtenida por el alumnado de cuarto curso respecto a los y las estudiantes de primero es únicamente una décima superior. Encontramos diferencias mayores entre hombres y mujeres (3,1 versus 3,4) y por grupos de edad, siendo 4 décimas inferior, con un 3,2, el promedio del estudiantado de entre 18 y 24 años.

En conjunto, sumadas todas las puntuaciones de los ítems del apartado en función del sentido positivo o negativo de las afirmaciones, observamos que se obtiene una puntuación promedio de 20,5, de un total de 30 puntos y un mínimo de 5 . Observamos que la mayor diferencia se da en una comparación entre sexos $-1,7$ puntos-, con una puntuación de 21,5 en ellas y un 19,8 en ellos, mientras que por cursos solo encontramos una diferencia de 7 décimas a favor de los de cuarto curso. Por último, el grupo de edad igual o mayor de 25 años obtiene 21,2 puntos, 8 décimas más que el de menores de 25 años.

\subsection{Percepción y conocimiento acerca de las mujeres y el trabajo en época romana}

En este segundo apartado nos centramos en aquellas afirmaciones que se refieren a aspectos relacionados con el trabajo de las mujeres en Roma. Se trata de cuestiones sobre las que las fuentes clásicas aportan generalmente escasa información, con algunas excepciones, como sucedería con la presencia pública de las mujeres en el ámbito religioso o, en cierta medida, con la imagen tradicionalmente establecida de la mujer romana como hiladora y tejedora. En todo caso, son aspectos que, salvo excepciones, han sido objeto de escasa atención por parte de la historiografía y que tampoco tienden a incluirse en la formación universitaria, aunque, dada su importancia, resulta de gran interés aproximarnos a la percepción del alumnado acerca de ellos (Figura 3).

Si en algún ámbito de la sociedad romana las mujeres tuvieron un espacio público reconocido, habría sido en la esfera religiosa, pudiendo integrar algunos colegios sacerdotales, como es el caso de las flamínicas o, en especial, el de las vestales, que se situaron entre las figuras más destacadas y conocidas de la religión oficial en Roma (Beard et al., 1998; Saquete Chamizo, 2000; Pavón Torrejón, 2015; Oria Segura, 2017). No obstante, ante la afirmación de que las mujeres romanas desempeñaban un papel público en la esfera religiosa, el alumnado mantiene posiciones considerablemente repartidas, aunque mostrando una mayor tendencia a aceptar el enunciado. Así, el 


\begin{tabular}{|c|c|c|c|c|c|}
\hline $\begin{array}{c}\text { Percepción y conocimiento } \\
\text { acerca de las mujeres y el trabajo } \\
\text { en época romana }\end{array}$ & 1-TED & 2-ED & $\begin{array}{c}3-\mathrm{Ni} \text { DA } \\
\text { ni ED }\end{array}$ & 4-DA & 5-TDA \\
\hline $\begin{array}{c}\text { 7. Las mujeres romanas desempeñaban } \\
\text { un papel público en la esfera religiosa. }\end{array}$ & $9,3 \%$ & $22,2 \%$ & $17,6 \%$ & $37,0 \%$ & $13,9 \%$ \\
\hline $\begin{array}{c}\text { 8. En la antigua Roma, las mujeres } \\
\text { podían llegar a ser grandes } \\
\text { propietarias y comerciantes. }\end{array}$ & $4,6 \%$ & $39,8 \%$ & $19,4 \%$ & $32,4 \%$ & $3,7 \%$ \\
\hline $\begin{array}{c}\text { 9. En la antigua Roma, hilar y tejer para } \\
\text { fabricar textiles habría sido realizado } \\
\text { mayoritariamente por mujeres. }\end{array}$ & $0,9 \%$ & $9,3 \%$ & $13,9 \%$ & $55,6 \%$ & $20,4 \%$ \\
\hline $\begin{array}{c}\text { 10. La producción cerámica en época } \\
\text { romana era mayoritariamente } \\
\text { realizada por mujeres. }\end{array}$ & $6,5 \%$ & $35,2 \%$ & $39,8 \%$ & $14,8 \%$ & $3,7 \%$ \\
\hline $\begin{array}{l}\text { 11. Parte de las pinturas murales y los } \\
\text { frescos romanos fueron pintados } \\
\text { por mujeres. }\end{array}$ & $4,6 \%$ & $34,3 \%$ & $47,2 \%$ & $13,9 \%$ & $0,0 \%$ \\
\hline
\end{tabular}
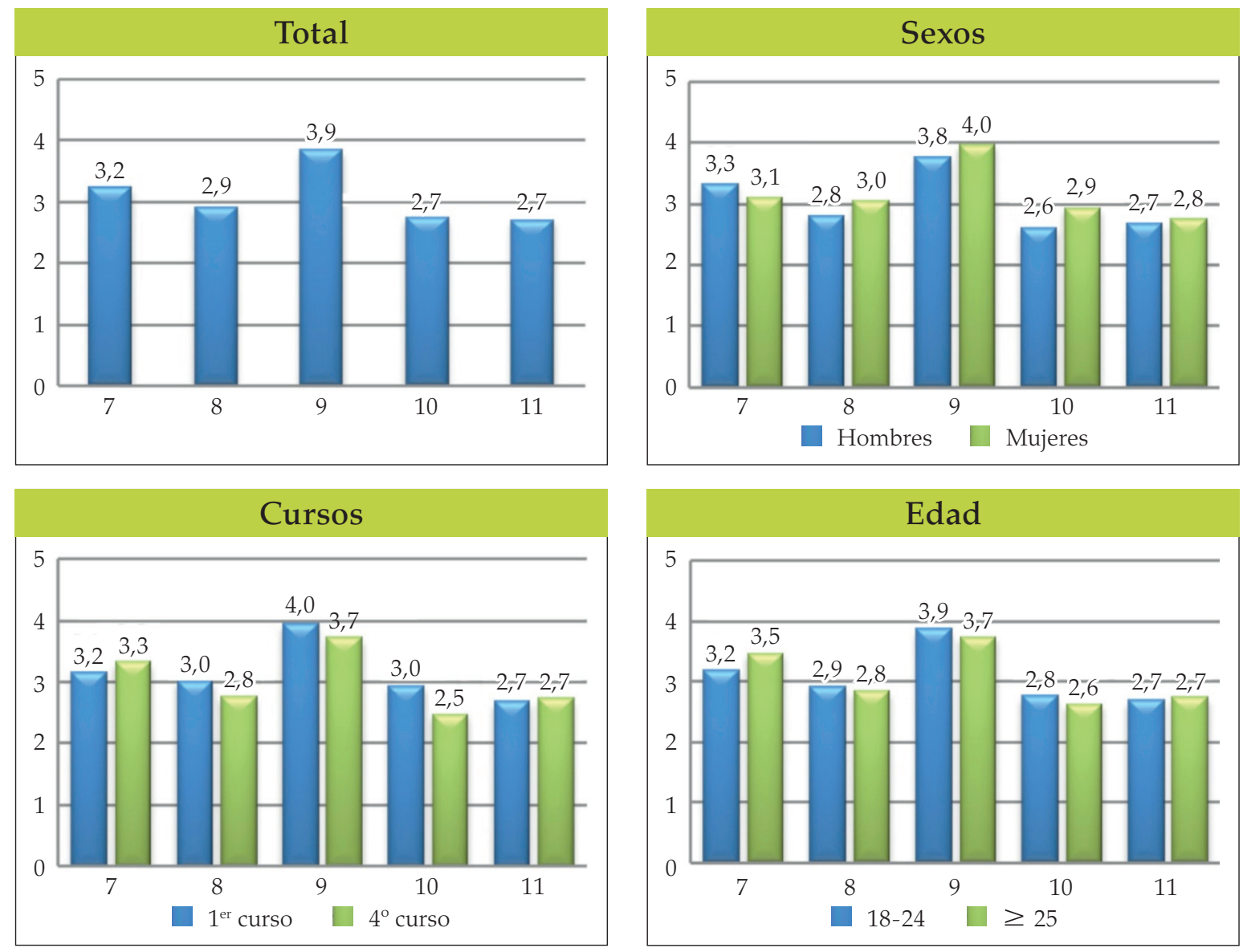

Figura 3

Ítems con porcentajes de respuesta y promedio por categorías (elaboración propia) 
31,5\% del estudiantado se pronuncia en desacuerdo o totalmente en desacuerdo, mientras que el 50,9\% se manifiesta de acuerdo o muy de acuerdo. El promedio conjunto se sitúa en el 3,2, con una diferencia leve de únicamente 2 décimas entre hombres y mujeres, con un 3,3 y un 3,1 respectivamente, mientras que el grupo de cuarto curso registra una única décima más que los de primero, siendo mayor la diferencia en función de la edad, con un 3,2 para los menores de 25 años y un 3,5 para los de 25 o superior.

Se observa un panorama notablemente diferente cuando se les cuestiona acerca de si las mujeres podían ser grandes propietarias y comerciantes, pues las posiciones, bastante equilibradas, se inclinan ligeramente hacia la negación. Las evidencias de la existencia de grandes propietarias y comerciantes a partir de época tardorrepublicana han sido recogidas por distintos trabajos (Berdowski, 2007; Alfaro Giner, 2010; Broekaert, 2013; Gregorio Navarro, 2015; entre otros), aunque esta posibilidad no estuviera reconocida en el marco jurídico (Pomeroy, 1995: 163; Berdowski, 2007: 285-286). Así, aunque hay un 32,4\% de acuerdo y un 3,7\% muy de acuerdo, el 39,8\% y el 4,6\% del alumnado se manifiesta en desacuerdo y totalmente en desacuerdo acerca de este planteamiento. Esta situación se registra también en el promedio total, que se sitúa ligeramente por debajo del 3, sin que se observen diferencias superiores a las 2 décimas en ninguna de las categorías analizadas.

Por el contrario, una gran mayoría del estudiantado $-76 \%$ - se manifiesta de acuerdo o muy de acuerdo con que el hilado y el tejido habrían sido actividades eminentemente desarrolladas por mujeres durante el periodo romano. Aunque no pueda considerarse que en época romana hubiera sido una tarea exclusivamente realizada por mujeres (Pomeroy, 1995: 199; Salisbury, 2001: 367; Berdowski, 2007: 290-291), la producción de tejidos ha sido reconocida como un trabajo femenino por la historiografía (Alfaro Giner, 1997; Fernández Uriel, 2011: 395; Medina Quintana, 2009), con evidencias en la epigrafía, la iconografía y los textos clásicos que apuntan en este sentido (Col. 12, 3; D. 33, 7, 12; Liv. 1, 57, 9; Suet, Aug. 64, 2). No obstante, debemos ser conscientes del posible sesgo de las fuentes dada la asociación de este trabajo con el modelo tradicional de mujer en el mundo grecolatino ${ }^{6}$. En este sentido, solo el 10,2\% de los alumnos y alumnas se muestra en desacuerdo, lo que se refleja en el promedio total alcanzado, que se sitúa en el 3,9. Se registra un posicionamiento ligeramente mayor a favor de esta afirmación por parte de las mujeres, con un promedio de 4 respecto al 3,8 de los hombres, mientras que entre el alumnado de primero y el de cuarto, la diferencia es de 3 décimas, con 4 y 3,7 puntos respectivamente.

En los dos últimos ítems que abordamos aquí destaca la gran cantidad de estudiantes que se manifiestan «ni de acuerdo ni en desacuerdo», lo que en gran parte

\footnotetext{
Para el caso de Roma sirva el ejemplo de la legendaria Lucrecia, utilizada durante siglos como modelo ideal de matrona romana, de la que Tito Livio $(1,57,9-10)$ nos cuenta que se encontraba tejiendo la lana mientras esperaba a su marido, antes de ser violada por el hijo de Tarquinio, en el famoso episodio que acabó con la expulsión del tirano y la implantación de la República (Liv. 1, 58, 5; 59, 3).
} 
puede ser debido a que inquieren por temas poco abordados en la historiografía y en las aulas. La primera de estas afirmaciones atribuye la producción cerámica de forma mayoritaria a las mujeres romanas, en contra de lo habitualmente reflejado en la literatura científica, sobre todo para su producción en serie, a pesar de que hay evidencias del desempeño por parte de mujeres en distintas esferas de este ámbito productivo (Salisbury, 2001: 368; Berdowski, 2007: 287-290; Alfaro Giner, 2010: 32; Medina Quintana, 2014). El 39,8\% del alumnado encuestado opta por la posición intermedia, aunque hay una tendencia a negar este planteamiento. Así, mientras que el 41,7\% afirma estar en desacuerdo o totalmente en desacuerdo, únicamente el 18,5\% declara estar de acuerdo o muy de acuerdo, lo que sitúa el promedio total en el 2,7. Destaca la significativa diferencia alcanzada entre el alumnado de primer curso y el de cuarto, pues aquel obtiene un promedio de 3 puntos, mientras que el de cuarto obtiene 2,5. También es interesante observar que un mayor número de alumnas suscribirían que la producción cerámica sería una actividad desempeñada sobre todo por mujeres, con 2,9 puntos, mientras que en el caso de los alumnos varones se obtienen 2,6.

Valores similares se han recogido al plantear al alumnado que detrás de parte de las pinturas murales y frescos romanos hubiera un trabajo desempeñado por mujeres, situándose este en una posición intermedia, ni de acuerdo ni en desacuerdo, con el 47,2\%, el mayor porcentaje alcanzado con ese posicionamiento en todos los ítems abordados en este artículo. Entre el resto de alumnas y alumnos hay una clara inclinación hacia el rechazo de este supuesto, con un 34,3\% en desacuerdo y un $4,6 \%$ totalmente en desacuerdo, mientras que solo el 13,9\% se manifiesta de acuerdo, siendo el único caso de la totalidad del cuestionario en el que nadie se ha posicionado totalmente de acuerdo. De igual modo, el promedio se sitúa en el 2,7, sin que haya apenas diferencias en función del curso, el sexo o la edad. Los resultados de este ítem son bastante rotundos, en tanto que el rechazo al ítem se da a pesar de que solo se plantea la posibilidad de que una parte de esa actividad fuese hecha por mujeres, no que fuese de forma mayoritaria ni en exclusiva. Acerca de esta cuestión, puede decirse que la información disponible sobre quienes ejecutaron pinturas en época romana es escasa, habiéndose considerado que la pintura parietal sería realizada por artesanos en talleres y generalmente de forma anónima (Abad Casal, 1982: 417, 453). Aunque existen algunas referencias acerca de mujeres pintoras en la Antigüedad (Plin Nat. 35, 147-148; Kampen, 1975; Salisbury, 2001: 21), de forma general, puede afirmarse que el tema se enfoca en masculino.

Observando de forma conjunta las cinco afirmaciones de este apartado a través de la puntuación total alcanzada vemos que, de un máximo de 25 puntos, el promedio se sitúa en 16,5. El promedio de la puntuación es 7 décimas superior en el grupo de edad igual o mayor de 25 años, que obtiene 17,1 puntos, respecto a los 16,4 que alcanza el de menores de 25 años. Es muy interesante señalar que las diferencias en función del sexo y el curso son, generalmente, leves. 


\section{VALORACIÓN}

Esta investigación ha proporcionado unos resultados que permiten hacer distintas valoraciones de interés. La primera de ellas es la obtención de una serie de promedios globales que, en su mayoría, revelan disparidad, inseguridad y/o desconocimiento acerca de la mayor parte de las cuestiones abordadas.

Así, cabe destacar resultados reveladores, como que un 32,4\% del alumnado encuestado de los estudios universitarios en Historia, sorprendentemente, no ha suscrito que el Imperio romano nunca tuvo a una mujer al frente. Asimismo, que el resultado global ante la afirmación de que las mujeres romanas no tenían derecho al voto no supera ni una décima la barrera del «de acuerdo» -4 puntos-, no alcanzándola en el caso de los alumnos varones y con casi un 15\% del estudiantado que no reconocería esta exclusión de las mujeres de las votaciones. En la antigua sociedad romana, el gobierno de la esfera pública y la ostentación de cargos en la política y la administración, no solo se consideraban algo propio de los hombres, sino que estaba prohibido a las mujeres ejercerlos (D. 50, 17, 2. pr. [Ulp. 1 Sab.]; Bauman, 1992; Alfaro Giner, 2010: 16; Gallego Franco, 2015: 315). A pesar de ello, las respuestas del alumnado parecen reflejar, como mínimo, una falta de claridad en la adquisición de conocimientos en esta línea -o quizá, también, de seguridad a la hora de reconocerlos y reproducirlos-. En todo caso, es importante destacar que una clara mayoría del alumnado acepta definir a Roma como una sociedad patriarcal, con más del 81\%.

A pesar de que la antigua Roma es uno de los periodos históricos en los que más aspectos se conocen sobre las mujeres (Cid López, 2015b), considerando lo apuntado por este estudio, una parte importante del conocimiento que se posee sobre la antigua sociedad romana no se reflejaría necesariamente en lo adquirido en las aulas. Así, por ejemplo, más del $48 \%$ del estudiantado no ha validado que el principal rol social atribuido a las mujeres de la élite en la sociedad romana fuera la reproducción. Estos resultados pueden llamar la atención, ya que podría considerarse como bastante conocida, sobre todo en el ámbito universitario de los estudios en Historia, la asociación entre las mujeres en la Antigüedad clásica -principalmente de la élite- y el espacio doméstico, en contraposición al espacio público, vinculado a los hombres (Casamayor Mancisidor, 2015a: 11), aunque en época romana esta división se haya considerado menos rígida que en la antigua Grecia ${ }^{7}$. Además, más del 49\% no ha dado como válido que las mujeres romanas desempeñaran un papel en la esfera pública a través de la religión, sin que la afirmación plasmada en el cuestionario calificara de más o menos influyente o determinante dicho papel, lo que podría ser objeto de debate.

Por otra parte, casi el 50\% del alumnado encuestado no consideraría que las mujeres en la Roma antigua tuvieran acceso a la educación y casi el 64\% no han afirmado que pudieran llegar a ser comerciantes y grandes propietarias. De acuerdo

7 Véase, por ejemplo, el reciente trabajo de Beard (2017). 
con diversos tipos de fuentes de información histórica, mujeres durante la época romana figuran como propietarias de tierras, así como asociadas a la producción y comercialización de productos como el cereal, el vino y el aceite (Berdowski, 2007: 292; Alfaro Giner, 2010: 22, 30; Fernández Uriel, 2011: 386, 391), la lana (Alfaro Giner, 1997; Medina Quintana, 2009), o los ladrillos y cerámicas, tanto como propietarias o comercializadoras, como también en lo referente al trabajo manual (Salisbury, 2001: 368; Berdowski, 2007: 287-288; Alfaro Giner, 2010: 32; Medina Quintana, 2014).

En este sentido, uno de los aspectos más desconocidos, no solo para el periodo romano, sino de manera transversal para otras muchas épocas históricas, es el de la relación entre las mujeres y el trabajo, lo que ha motivado en buena parte la inclusión de ítems en relación con este tema en el presente estudio. El trabajo realizado por las mujeres en la Antigüedad aparece concebido en las fuentes escritas como de escaso valor, respecto al realizado por los hombres (Alfaro Giner, 2010: 16), al igual que en la mayor parte de las sociedades conocidas (Hartmann, 1980), al estar las propias mujeres socialmente valoradas de manera inferior a los hombres (Cobo Bedia, 1995). No obstante, a pesar del desconocimiento general existente en este sentido, de acuerdo con la información disponible, las mujeres romanas ejercieron muy diversos trabajos y profesiones, por ejemplo, como médicas, comadronas, escribientes, comediantes, empleadas en minas, e incluso gladiadoras (Alfaro Giner, 2010: 20; Medina Quintana, 2014; Gregorio Navarro, 2015: 277; Ortega Balanza, 2017), aspectos de la Roma antigua que no suelen conocerse. A este desconocimiento contribuye que el trabajo de las mujeres no fuera apenas reflejado en los textos o en otras representaciones de la propia época romana, como por ejemplo se observa en los relieves funerarios y decorativos (Picazo-Gurina, 2017).

Por otro lado, en el marco de este mismo proyecto de investigación enfocado al análisis de la percepción social y el conocimiento del alumnado del Grado en Historia acerca de la desigualdad entre hombres y mujeres en el pasado, pudimos comprobar, en referencia a otras cuestiones generales planteadas al estudiantado (Pastor Quiles y Mateo Corredor, 2018), que las variaciones en las respuestas de hombres y mujeres eran en su mayoría leves, siendo mayores las mostradas en función del curso. En cambio, en este caso, el de los ítems referidos a la Roma antigua, de naturaleza algo distinta a la de otras afirmaciones planteadas en el mismo proyecto, las reacciones del estudiantado han proporcionado también resultados diferentes, permitiendo distinguir otras tendencias en las respuestas.

Observando ambos grupos de proposiciones, en el primero, relativo a la desigualdad política y jurídica, las mayores diferencias encontradas han sido en función del sexo, siendo esta diferencia más de dos veces superior que en una comparación entre el estudiantado de primero y de cuarto curso, lo que es, sin duda, significativo. En el segundo grupo, referido a las mujeres y las actividades económicas, las diferencias entre las respuestas de alumnas y alumnos son muy pequeñas, pero también lo son en función del curso, con pocas o ninguna décima de diferencia, salvo en el caso 
de la proposición referida a la producción cerámica como mayoritariamente femenina, rechazada por el alumnado de cuarto curso con medio punto más que en el de primero. Junto con la afirmación de que nunca hubo una mujer en la más alta magistratura del imperio, estas son las únicas proposiciones aquí estudiadas en las que el alumnado de cuarto muestra diferencias significativas respecto al que comienza sus estudios.

No obstante, se vislumbra una cierta tendencia en las alumnas a un mayor reconocimiento de las condiciones de desigualdad de las mujeres en la sociedad romana antigua. Así, por ejemplo, se muestran más de acuerdo que los alumnos varones en que el rol principal socialmente atribuido a las mujeres de la élite romana era la reproducción -con una diferencia en las respuestas muy destacada en una comparación entre sexos, de casi 1 punto-, con la exclusión de las mujeres del voto o con la ausencia de mujeres en el cargo de emperador. Además, en el caso de otras proposiciones en las que se observan diferencias en función del sexo, las alumnas se aproximan ligeramente más a la realidad apuntada por la investigación en comparación con los alumnos varones -con una diferencia de 2-3 décimas-, como en el caso del reconocimiento de que las mujeres romanas podían llegar a tener acceso al divorcio, la concepción de la actividad textil como un trabajo eminentemente femenino y el reconocimiento de que las mujeres podían ser comerciantes y grandes propietarias en la antigua Roma. En cambio, los alumnos varones se muestran más en desacuerdo que las alumnas con que la producción cerámica en el periodo romano fuera mayoritariamente realizada por mujeres y en que detrás de la pintura parietal romana hubiera participación femenina, aunque en ambas cuestiones el promedio del alumnado se muestra en desacuerdo y las diferencias mostradas entre sexos son también de pocas décimas.

En definitiva, los resultados de esta investigación parecen apuntar a que los temas aquí tratados referidos a la sociedad romana serían, al menos en parte, poco abordados en los contenidos de la titulación, lo que se vería reflejado en que apenas se aprecien cambios entre las reacciones del alumnado que comienza y el que termina sus estudios en Historia. Referidos a la desigualdad entre sexos en el ámbito más directamente político, en el jurídico o a las actividades económicas, entre las cuestiones planteadas se encuentran aspectos fundamentales que contribuirían a definir a la sociedad patriarcal romana. Esta, como parece verse reflejado también en este estudio a través de la indefinición o el desconocimiento plasmado en las respuestas, ha sido tradicionalmente abordada, como mínimo, sin visibilizar y hacer explícitas las formas que tomó en la sociedad romana la dominación de las mujeres, así como sus mecanismos de legitimación.

Si en el marco del conocimiento androcéntrico mayoritariamente establecido y transmitido sobre la antigua Roma parece no insistirse apenas en la materialización 
de la desigualdad entre hombres y mujeres -como la exclusión por ley del sexo femenino en la política o en el derecho-, menos se visibilizan todavía las maneras de normalizar y reproducir esa desigualdad entre sexos. Valga como ejemplo la legitimación de la violencia contra las mujeres inherente al patriarcado romano (Gregorio Navarro, 2015: 271), con formas brutales de control social de las mujeres, como el culto femenino a Tacita $\mathrm{Muta}^{8}$, conmemoración de un símbolo de la necesaria censura de la palabra de las mujeres y de los castigos correctivos que puede suponer para las mujeres romanas «hablar de más» (Casamayor Mancisidor, 2015a: 12; 2015b).

El conocimiento acerca de la desigualdad entre hombres y mujeres en la Roma antigua o, dicho de otro modo, del patriarcado romano, es imprescindible, ya que este impregna multitud de ámbitos de la sociedad romana, como de la Antigüedad en general, que han sido y son hoy en día ampliamente objeto de estudio. Así, están muy presentes, no solo en el ámbito académico, sino también en la sociedad en general, dada la gran trascendencia social de la Historia Antigua y, en especial, de la Historia de Roma. Está en las manos de las y los profesionales de la Historia continuar contribuyendo al conocimiento de estas desigualdades y acabar así, poco a poco, con el silencio en torno a ellas del que han ido históricamenteacompañadas.

8 Esta náyade se habría atrevido a tratar de defender a otra mujer de la violencia sexual de Júpiter, arrancándole este la lengua y enviándola al inframundo, de camino al cual es violada por Mercurio sin poder emitir un grito. De este terrorífico episodio nacieron los Lares, de acuerdo con Ovidio (Fast. II, 585-615), véase Casamayor Mancisidor (2015b). Sobre otras formas de silenciamiento de las mujeres en la Antigüedad, véase Beard (2017). 


\section{REFERENCIAS BIBLIOGRÁFICAS}

ABAD CASAL, L. (1982), La pintura romana en España, tomo 1, Universidad de Alicante Universidad de Sevilla, Sevilla.

ALFARO GINER, C. (1997), El tejido en época romana, Arco Libros, Madrid.

- (2010), «La mujer y el trabajo en la Hispania prerromana y romana. Actividades domésticas y profesionales», Mélanges de la Casa de Velázquez, 40, 2, 15-38.

BAUMAN, R. A. (1992), Women and politics in Ancient Rome, Routledge, London.

BEARD, M. (2017), Women \& power. A manifesto, Profile Books, London.

BEARD, M.; NORTH, J. y PRICE, S. (1998), Religions of Rome. Vol. I. A History, Cambridge University Press, Cambridge.

BENKE, N. (2012), «On the Roman father's right to kill his adulterous daughter», The History of the Family, 17, 284-308.

BERDOWSKI, P. (2007), «Some remarks on the economic activity of women in the Roman Empire: A research problem», en P. Berdowski y B. Blahaczek, Haec mihi in animis vestris templa. Studia classica. In memory of Professor Lestaw Morawiecki, 283-298, Institute of History at The University of Rzeszów-The Rzeszów Archaeological Foundation, Rzeszów.

BRAVO BOSCH, Ma J. (2011), «El proceso electoral en Roma: la publicidad política en la época tardorrepublicana», Revista jurídica Universidad Autónoma de Madrid, 23, 13-33.

BROEKAERT, W. (2013), Navicularii et negotiantes: a prosopographical study of Roman merchants and shippers, Pharos: Studien zur griechisch-römischen Antike 28, Verlag Marie Leidorf, Rahden/Westf.

CANTARELLA, E. (2016), «Women and Patriarchy in Roman Law», en P. Du Plessis, C. Ando y K. Tuori (eds.), The Oxford Handbook of Roman Law and Society, 419-431, Oxford University Press, Oxford.

CASAMAYOR MANCISIDOR, S. (2015a), «Casta, pia, lanifica, domiseda: Modelo ideal de feminidad en la Roma tardorrepublicana (ss. II-I a. C.)», Ab Initio, 11, 3-23.

- (2015b), «Tacita Muta y el silencio femenino como arma del patriarcado romano», Panta Rei. Revista Digital de Ciencia y Didáctica de la Historia, 5, 27-41.

CASTÁN PÉREZ-GÓMEZ, S. (2013), «Una aproximación a la Democracia Romana a través del Sufragio Activo y Pasivo», en A. Valmaña Ochaíta (coord.), Democracia en el mundo antiguo y en la actualidad, 55-132, Andavira, Santiago de Compostela.

CID LÓPEZ, R. Ma (2001), «La educación de la niña romana: de puella a matrona docta», en V. Alfaro y R. Francia, Bien enseñada. La formación femenina en Roma y el Occidente romanizado, 19-44, Servicio de Publicaciones y Divulgación Científica de la Universidad de Málaga, Málaga.

- (2015a), «El género y los estudios históricos sobre las mujeres de la Antigüedad. Reflexiones sobre los usos y evolución de un concepto», Revista de Historiografía, 22, 25-49.

- (2015b), «Las silenciosas mujeres de la Roma antigua. Revisiones desde el género y la Historia», en A. Domínguez Arranz y R. Ma Marina Sáez, Género y enseñanza de la Historia, 187-212, Sílex, Madrid. 
COBO BEDIA, R. (1995), «Género», en C. Amorós Puente (dir.), Diez palabras clave sobre mujer, 55-83, Verbo Divino, Estella.

CONESANAVARRO, P. D. (2016), «Estudios sobre mujeres de la Antigua Roma. Estado de la cuestión, problemas y progreso científico en un campo histórico consolidado», Minius, 24, 205-226.

FABILA ECHAURI, A. Mª MINAMI, H. e IZQUIERDO SANDOVAL, M. J. (2013), «La escala de Likert en la evaluación docente: acercamiento a sus características y principios metodológicos», Perspectivas docentes, 50, 31-40.

FARRELL, A. D.; RABINOWITZ, J. A.; WALLANDER, J. L. y CURRAN, J. P. (1985), «An evaluation of two formats for the intermediate-level assessment of social skills», Behavioral Assessment, 7 (2), 155-171.

FAYER, C. (2005), La Familia Romana: Aspetti Giuridici ed Antiquari. Concubinato Divorzio Adulterio. Parte Terza, L'Erma di Bretschneider, Roma.

FERNÁNDEZ URIEL, P. (2011), «Obreras y empresarias en el Periodo Romano Alto Imperial», Espacio, Tiempo y Forma, Serie II, Historia Antigua, 24, 367-390.

GALLEGO FRANCO, H. (2015), «Crónicas de una relación ilícita. Mujeres, acción política e historiografía en Hispania tardoantigua», en A. Domínguez Arranz y R. Ma Marina Sáez, Género y enseñanza de la Historia, 315-343, Sílex, Madrid.

GARCÍA SANTA MARÍA, T. y PAGÈS BLANCH, J. (2008), «La imagen de la Antigüedad en la enseñanza de la Historia», en $\mathrm{M}^{\mathrm{a}} \mathrm{J}$. Castillo Pascual (coord.), Congreso Internacional «Imagines». La Antigüedad en las Artes escénicas y visuales, 691-720, Logroño 22-24 de octubre de 2007, Universidad de La Rioja, Logroño.

GEORGE, D. y MALLERY, P. (2003), Spss for Windows step by step: A Simple Guide and Referece. 11.0 Update (4. ${ }^{a}$ ed.), Allyn \& Bacon, Boston.

GREGORIO NAVARRO, Ma C. D. (2015), «Violencia de género y privación de libertad en el mundo clásico», en A. Domínguez Arranz y R. Ma Marina Sáez, Género y enseñanza de la Historia, 269-290, Sílex, Madrid.

HARTMANN, H. (1980), «Un matrimonio mal avenido: hacia una unión más progresiva entre marxismo y feminismo», Zona Abierta, 24, 85-113.

HEMELRIJK, E. A. (1999), Matrona docta. Educated women in the roman elite from Cornelia to Julia Domna, Routledge, London/New York.

KAMPEN, N. B. (1975), «Hellenistic artists: Female», Archeologia Classica, 27, 9-17.

LEVICK, B. (2012), «Women and Law», en S. L. James y S. Dillon, A companion to women in the ancient world, 96-106, Wiley-Blackwell, Chichester.

LÓPEZ MEDINA, Ma J. (2008), «El trabajo de las mujeres en las unidades domésticas campesinas del Sureste peninsular durante el Alto Imperio romano», en T. Escoriza Mateu, Ma J. López Medina y A. Navarro Ortega (eds.), Mujeres y arqueología. Nuevas aportaciones desde el materialismo histórico, 199-252, Junta de Andalucía, Sevilla. 
MEDINA QUINTANA, S. (2009), «Las mujeres y la producción textil en la Roma Antigua», Cuestiones de género: de la igualdad y la diferencia, 4, 51-64.

- (2014), Mujeres y economía en la Hispania romana: oficios, riqueza y promoción social, Trabe, Oviedo.

MORENO SECO, M. (2014), Historia de las Mujeres y de Género en los estudios de Grado, http://aeihm.org/historia/historia-mujeres-genero-estudios-grado (consultado el 20 de enero de 2019).

NAMAKFOROOSH, M. N. (2000), Metodología de la investigación, Limusa, México.

ORIA SEGURA, M. (2017), «Mujeres y religión en el mundo romano: enfoques cambiantes, actitudes presentes», Arenal, 24 (1), 73-98.

ORTEGA BALANZA, M. (2017), «La violencia como constructor de identidad en la gladiatura femenina», en B. Antela-Bernárdez, C. Zaragozà Serrano y A. Guimerà Martínez (eds.), Placer y Dolor. Las mujeres en la Antigüedad, Monografías, 72, Universidad de Alcalá de Henares, Madrid.

PASTOR QUILES, M. y MATEO CORREDOR, D. (2018), «La desigualdad entre mujeres y hombres, en el pasado y en el presente. Un estudio preliminar sobre su percepción y conocimiento en el alumnado de Historia», El Futuro del Pasado, 9, 365-391.

PAVÓN TORREJÓN, P. (2015), «La mujer en la religión romana: entre la participación y la marginación», en E. Ferrer y Á. Pereira (coords.), Hijas de Eva. Mujeres y Religión en la Antigüedad, 115-141, Universidad de Sevilla, Sevilla.

PICAZO GURINA, M. (2017), «Más allá de los estereotipos: nuevas tendencias en el estudio del género en arqueología clásica», Arenal, 24 (1), 5-31.

POMEROY, S. B. (1995) [1975], Goddesses, Whores, Wives, and Slaves: Women in Classical Antiquity, Schocken Books, New York.

SALISBURY, J. E. (2001), Encyclopedia of women in the ancient world, ABC-CLIO, Santa Barbara.

SAQUETE CHAMIZO, J. C. (2000), Las vírgenes vestales: Un sacerdocio femenino en la religión pública, Anejos de Archivo Español de Arqueología 21, Madrid.

SERRANO LOZANO, D. (2012), «Cine y Antigüedad: pasado y presente en la pequeña y gran pantalla», Historia Autónoma, 1, 37-52.

SIERRA BRAVO, R. (2001), Técnicas de Investigación Social teoría y ejercicios, Paraninfo, Madrid.

URBANIK, J. (2016), «Husband and Wife», en P. Du Plessis, C. Ando y K. Tuori (eds.), The Oxford Handbook of Roman Law and Society, 473-486, Oxford University Press, Oxford. 